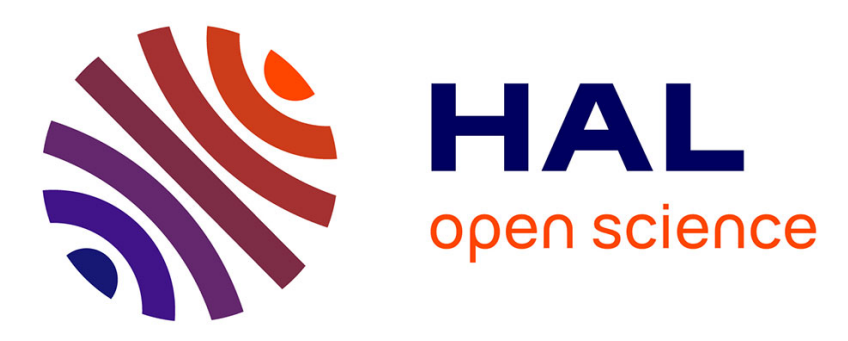

\title{
Defect-controlled hypersound propagation in hybrid superlattices
}

\author{
D. Schneider, F. Liaqat, E.H. El Boudouti, O. El Abouti, W. Tremel, H.J. \\ Butt, Bahram Djafari-Rouhani, G. Fytas
}

\section{- To cite this version:}

D. Schneider, F. Liaqat, E.H. El Boudouti, O. El Abouti, W. Tremel, et al.. Defect-controlled hypersound propagation in hybrid superlattices. Physical Review Letters, 2013, 111, pp.164301-1-5. 10.1103/PhysRevLett.111.164301 . hal-00879487

\section{HAL Id: hal-00879487 \\ https://hal.science/hal-00879487}

Submitted on 26 Aug 2021

HAL is a multi-disciplinary open access archive for the deposit and dissemination of scientific research documents, whether they are published or not. The documents may come from teaching and research institutions in France or abroad, or from public or private research centers.
L'archive ouverte pluridisciplinaire HAL, est destinée au dépôt et à la diffusion de documents scientifiques de niveau recherche, publiés ou non, émanant des établissements d'enseignement et de recherche français ou étrangers, des laboratoires publics ou privés. 


\title{
Defect-Controlled Hypersound Propagation in Hybrid Superlattices
}

\author{
Dirk Schneider, ${ }^{1}$ Faroha Liaqat, ${ }^{2}$ El Houssaine El Boudouti, ${ }^{3,4}$ Ossama El Abouti, ${ }^{3}$ Wolfgang Tremel, ${ }^{2}$ \\ Hans-Jürgen Butt, ${ }^{1}$ Bahram Djafari-Rouhani, ${ }^{4, *}$ and George Fytas ${ }^{1,5, \dagger}$ \\ ${ }^{1}$ Max Planck Institute for Polymer Research, Ackermannweg 10, 55128 Mainz, Germany \\ ${ }^{2} J o h a n n e s$ Gutenberg University, Duesbergweg 10-14, 55128 Mainz, Germany \\ ${ }^{3}$ LDOM, Département de Physique, Faculté des Sciences, Université Mohamed I, 60000 Oujda, Morocco \\ ${ }^{4}$ Institut d'électronique, de Microélectronique et de Nanotechnologie (IEMN), UMR-CNRS 8520, \\ UFR de Physique, Université de Lille 1, 59655 Villeneuve d'Ascq, France \\ ${ }^{5}$ Department of Materials Science, University of Crete and IESL/FORTH, 71110 Heraklion, Greece \\ (Received 17 August 2013; revised manuscript received 17 September 2013; published 14 October 2013)
}

\begin{abstract}
We employ spontaneous Brillouin light scattering spectroscopy and detailed theoretical calculations to reveal and identify elastic excitations inside the band gap of hypersonic hybrid superlattices. Surface and cavity modes, their strength and anticrossing are unambiguously documented and fully controlled by layer thickness, elasticity, and sequence design. This new soft matter based superlattice platform allows facile engineering of the density of states and opens new pathways to tunable phoxonic crystals.
\end{abstract}

DOI: 10.1103/PhysRevLett.111.164301

Selective transmission of elastic waves in superlattices (SLs), reported in 1979, has inaugurated the idea of phonon filtering by dielectric Bragg mirrors [1]. Fourteen years later, the study of phononic stop bands in 3D nanostructures was initiated by calculations of the full band structure of periodic composites [2], a followup of the electromagnetic analogs proposed in 1987 [3]. The necessity of combining materials of significantly different acoustic impedance in a periodically ordered assembly is the basis for phononic effects and has been exemplified both experimentally and theoretically [4-8]. SLs largely facilitate the study of phononic crystals, as the complex vector nature of the elastic wave propagation is subdued in one-dimensional structures. This platform allows the discovery of a new class of states and thereby the manipulation of elastic wave propagation in periodic structures. Longitudinal acoustic phonons folded to the Brillouin zone (BZ) [9] and confined acoustic vibrations in a phonon cavity of a SL embedded within an optical cavity [10] have been observed in semiconductor SL by Raman scattering. Defect modes, either surface localized within the frequency gaps of the zone folded phonons [11-13] or surface avoiding with wave vectors in the vicinity of the center [14] and edge of the BZ [15], have been revealed by Raman and pump-probe experiments. Many applications benefit from SL designs with controlled defects such as coherent phonon generation [16-19], concurrent modulation of light and sound [10,20-22], acoustic diodes [23], and reduction of heat conductivity [24,25].

However, to date these functional SLs are exclusively based on semiconductor materials with relatively low elastic impedance contrast and strong optical absorption preventing probing far from the surface, while their fabrication requires epitaxial growth under clean-room conditions. Instead, hybrid SLs comprising hard inorganics and
PACS numbers: 46.40.Cd, 63.20.D-, 68.55.am, 68.65.Cd

soft polymers pose an alternative flexible platform with distinct advantages: large and tunable band gaps [26-28], easy fabrication [29] and variation of total thickness and spacing for concurrent operation at hypersonic phonon and visible photon frequencies. Using these new soft matter based SLs, we present the first unequivocal observation of surface modes and their interaction with cavity modes varying the material and the thickness of the top layer, as well as the thickness and position of the cavity layer. The full theoretical description of both the experimental phononic band structure and the Brillouin light scattering (BLS) spectra is based on a Green's function method in the frame of elasticity theory, as concisely presented in the Supplemental Material [30]. This formalism directly accesses the density of states (DOS), which helps to identify the modes inside and near the edges of the 1st BZ that are activated through breaking of the high symmetry. The gained understanding is a precondition for reliable predictions of phonon propagation in periodic composite structures.

Hybrid SLs consisting of alternating poly(methyl methacrylate) (PMMA), and porous silica $\left(\mathrm{SiO}_{2}\right)$ layers were fabricated by high-speed spin coating on a glass substrate with PMMA forming the first layer. The thickness of each layer was adjusted by the concentration of the respective stock solutions (PMMA in toluene and aqueous dispersions of $\mathrm{SiO}_{2}$ nanoparticles). Heat treatment at $105^{\circ} \mathrm{C}$ finalizes each coating cycle with an unavoidable infiltration of PMMA into the $\mathrm{SiO}_{2}$ layer. The density and longitudinal sound velocity of PMMA $\left(\mathrm{SiO}_{2}\right)$ are $\rho=1190 \mathrm{~kg} \mathrm{~m}^{-3}$ $\left(\sim 1700 \mathrm{~kg} \mathrm{~m}^{-3}\right)$ and $c_{L}=2800 \mathrm{~m} \mathrm{~s}^{-1}\left(\sim 3150 \mathrm{~m} \mathrm{~s}^{-1}\right)$, respectively [26]. Fabrication, structure, and architecture of the investigated SLs are described in the Supplemental Material [30]. The periodicity ( $a$ ) of the SL, the thickness $\left(d_{c}\right.$ or $\left.d_{s}\right)$ and location $\left(\mathcal{N}^{P}\right.$ or $\mathcal{N}^{S}$ relative to the glass 
substrate) of cavity $(c)$ and surface $(s)$ layers ( $P$ for PMMA or $S$ for $\mathrm{SiO}_{2}$ ) are listed for seven supported SLs in Table I.

Spontaneous BLS, a nondestructive and noncontact optical technique, is employed to record the dispersion relation of longitudinal hypersonic $(\mathrm{GHz})$ excitations at different scattering wave vectors q [30]. Photoelastic interaction between single mode incident photons and thermally excited phonons give rise to momentum transfer $\mathbf{q}=\mathbf{k}_{\mathrm{s}}-\mathbf{k}_{\mathrm{i}}$ along a specified direction determined by the scattering geometry, with $\mathbf{k}_{\mathrm{s}}\left(\mathbf{k}_{\mathrm{i}}\right)$ being the wave vector of the scattered (incident) photons. The associated phonon energy is represented by the frequency shift $\pm \omega$ of the inelastically scattered light resolved by an actively stabilized tandem FP interferometer. Phonon wavelength and periodicity commensurability, i.e., $q a=O(1)$ enables access to the band diagram of the present SLs near the edge of the 1st BZ.

Hybrid SLs with uniform lattice spacing exhibit large band gaps for phonon propagation normal to the layers $[26,27]$. Structural disorder due to thickness fluctuations $(\sim 5 \%)$ was accommodated by the BLS instrumental width rendering the spectral pattern in the band gap region robust [27]. The theoretical spectra were convoluted with an instrumental function to represent the corresponding experimental BLS spectra. In this approach we can account for both the instrumental resolution and the conceivable weak hypersonic absorption in the hybrid SL structures. In fact, the acoustic attenuation, which can be described in the theory by using complex sound velocities for each sublayer, essentially causes broadening of the peaks in the DOS and hence the BLS spectra.

Insertion of defects into the hybrid SLs can, in principle, be realized by a manifold of different ways, e.g., variation of thickness and materials. Here, defected SLs are exemplified by surface and cavity layers of either constituent material (Table I). The control of boundary conditions is

TABLE I. Structure and architecture of hybrid SLs.

\begin{tabular}{lccccc}
\hline \hline & & \multicolumn{2}{c}{ Cavity $_{\text {layer }}{ }^{\mathrm{a}}$} & \multicolumn{2}{c}{ Surface $^{2}$ layer $^{\mathrm{a}}$} \\
ID & $a(\mathrm{~nm})$ & $d_{c}(\mathrm{~nm})$ & $\mathcal{N}^{\text {mat }}$ & $d_{s}(\mathrm{~nm})$ & $\mathcal{N}^{\text {mat }}$ \\
\hline SL1 & 98 & $\ldots$ & $\ldots$ & 57 & $17^{P}$ \\
SL2 & 100 & $\ldots$ & $\ldots$ & 80 & $17^{P}$ \\
SL3 & 97 & 62 & $9^{P}$ & 54 & $17^{P}$ \\
SL4 & 94 & 92 & $9^{P}$ & 44 & $17^{P}$ \\
SL5 & 94 & 140 & $9^{P}$ & 45 & $17^{P}$ \\
SL6 & 91 & 85 & $9^{P}$ & 85 & $18^{S}$ \\
SL7b & 93 & 100 & $2^{S}$ & $\ldots$ & $\ldots$ \\
& & 85 & $19^{P}$ & & \\
\hline
\end{tabular}

${ }^{\text {a }}$ Superscripts denote the material, e.g., PMMA $(P)$ or $\mathrm{SiO}_{2}(S)$ of the respective layers and the numbers indicate the position from the glass substrate. The thickness values are subject to $\pm 5 \%$ error.

${ }^{\mathrm{b}}$ See the Supplemental Material [30]. implemented by the choice of the surface layer. The SLs are terminated with either a hard $\mathrm{SiO}_{2}$ or soft PMMA layer that strongly impacts the band gap region as reported herein.

Figure 1 is a paradigm for surface-defect induced qualitative changes in the band diagram as illustrated for SL1 with eight bilayers (8 BL) and PMMA surface layer [Table I and Fig. 1(f)]. A new mode with frequency around $14 \mathrm{GHz}$ falls inside the band gap region of an infinite defect-free SL [27,28]; the solid lines in Fig. 1(a) represent theoretical calculations for an infinite SL. The small thickness of SL1 enables mode resolution in the BLS spectrum [Fig. 1(d)] as the separation between the three modes [DOS in Fig. 1(b)] exceeds the instrumental width $(\sim 0.5 \mathrm{GHz})$. Moreover, modes along the longitudinal acoustic (LA) branch are more intense than along the folded $\mathrm{FLA}^{-1}$ [27,31] as suggested by the contour plot in Fig. 1(a). Hence, suitable $q$ values have been selected for a strong detection [white circles in Fig. 1(a)]. The optimal design of the present SL was based on theoretical simulations of the BLS spectra and the band diagram: thinner SLs, e.g., with 5 BL would reduce the BLS signal and the sampling quality of the dispersion relation [Fig. 1(g)]; thicker SLs (20 BL) would decrease the separation of the individual modes rendering their experimental resolution hard [Fig. 1(h)]. These finite size effect calculations for SLs have been reported by some of the authors earlier [7]. Some of these modes are observed as sets of small satellite lines in high-resolution Raman spectra [32-34]. However, this is the first experimental documentation utilizing the advantages of hybrid SLs and coherent BLS.

The total DOS for SL1 in Fig. 1(b) reveals three main contributions, the upper $\left(e_{2}\right)$ and lower $\left(e_{1}\right)$ edge modes, and the surface mode $(s)$, whose nature can be identified by their displacement fields [Fig. 1(e)]. This mode assignment is further supported by the pattern of the local DOS at the surface [Fig. 1(c)]. It should be noted that the peak in the DOS associated with this $s$ mode becomes narrower as the number of BL increases; the coupling strength of the $s$ mode with the substrate modes weakens with surfacesubstrate separation. Hence, after convolution of this $s$ peak with the instrumental function, its documentation in the experimental BLS spectrum is severely affected. In fact, the contour plots for the notional SLs in Fig. 1(g) (5 BL) and Fig. 1(h) (20 BL) indicated by the $s$ arrows demonstrates the suppression of the $s$ mode with the SL thickness.

The local DOS at the surface [Fig. 1(c)] underlines the importance of the $s$ mode, while the $e$ modes have their maximum displacement centered in the middle of the SL [Fig. 1(e)]. Moreover, the envelope function of the displacement field for mode $e_{2}$ almost vanishes at the surface. The latter can therefore be qualified as a surface avoiding mode (SAM) recently reported for semiconductor SLs $[15,35]$. Additional evidence of the surface mode is its 
(a)

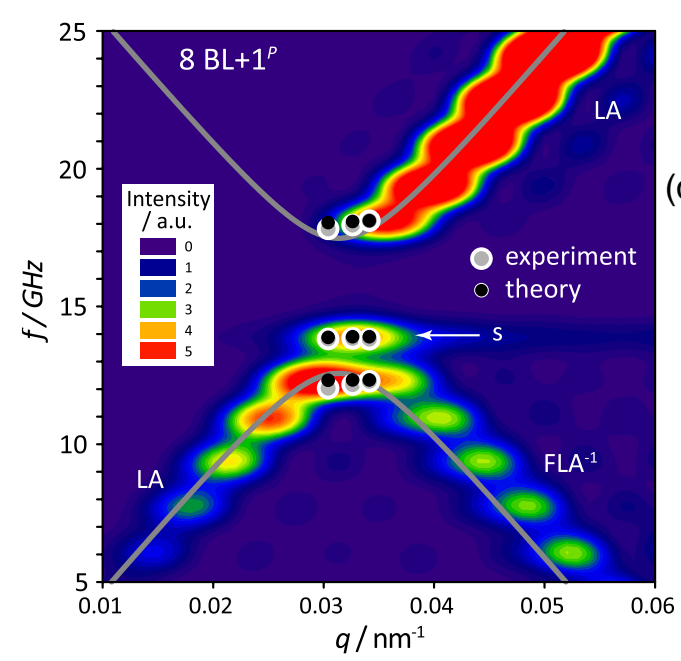

(f)

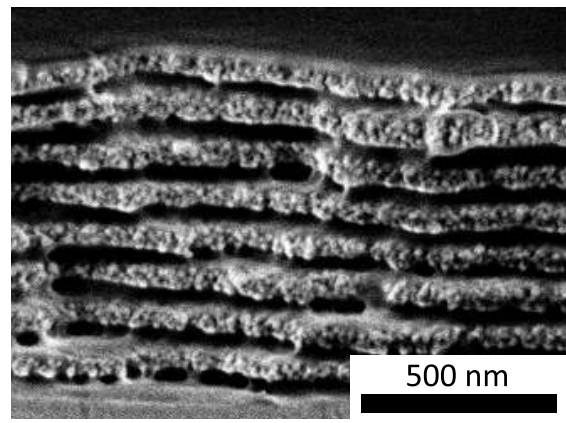

(b)

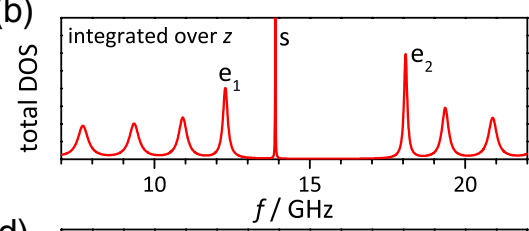

(d)

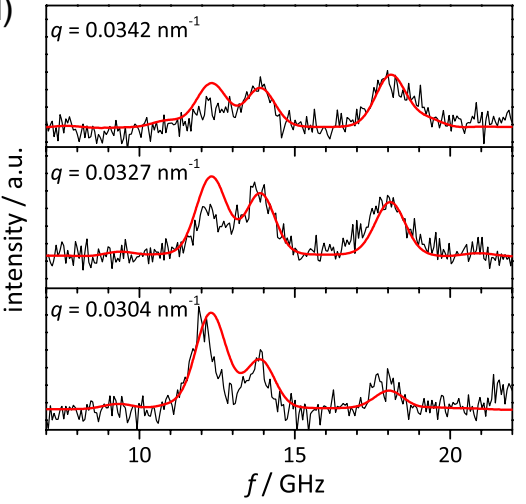

(g)

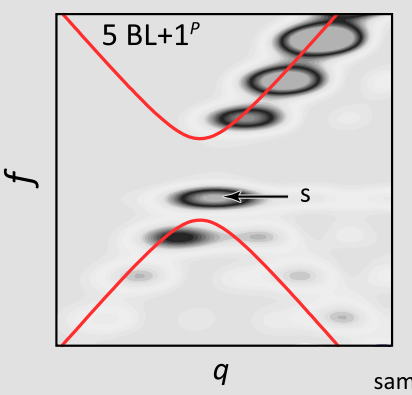

(c)

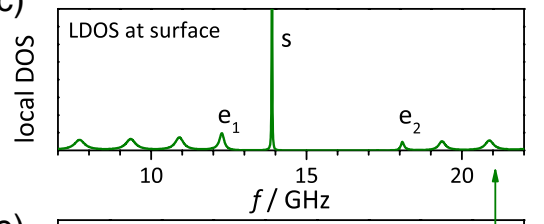

(e)

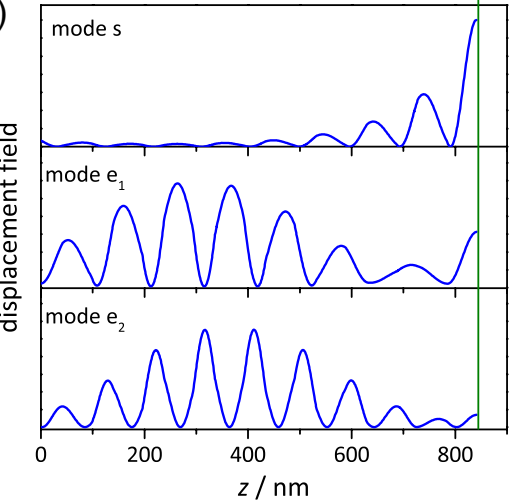

(h)

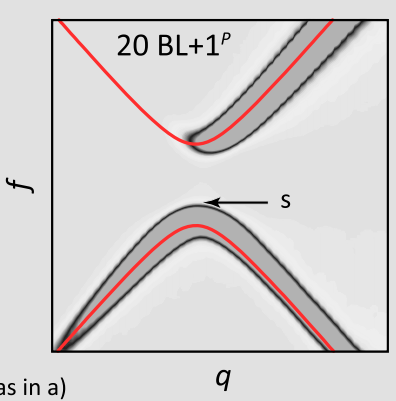

FIG. 1 (color online). (a) Dispersion relation for SL1 (Table I) with 8 bilayers (BL) and a PMMA surface layer $\left(17^{P}\right)$. The theoretical Brillouin intensity is given as a color scale and the dispersion of an infinite SL as solid lines. Peak positions of experimental (theoretical) spectra are given as white (black) circles. (b) Total density of states and local DOS at the surface (c) with indicated edge $e_{1,2}$ and surface modes $s$. (d) Experimental (theoretical) Brillouin spectra in black (red solid line) at three $q$ values. (e) Displacement fields of the modes indicated in (c). (f) Cross sectional electron micrograph of SL1. (g) and (h) Mode separation and strength of the surface mode $s$ in SLs with $5 \mathrm{BL}$ (g) and $20 \mathrm{BL}(\mathrm{h})$; mode $s$ (indicated by arrows) is hardly discernible in (h).

dependence on the top layer thickness $[7,36]$. Theoretically [7], the frequency of mode $s$ should decrease with $d_{s}$ and its frequency could then be easily tuned inside the gap.

In fact, SL2 (Table I) nicely exemplifies this situation as demonstrated by the good agreement with the theoretical predictions in Fig. 2. The upper band edge mode behaves as a SAM, whereas the lower edge mode is merged with a surface mode close to the lower band (solid line) and decays slowly into the SL.

Insertion of cavity layers in the interior of the SLs as schematically shown in Fig. 3(c) represents a second class of defects distinctly manifested in the band diagram. Additionally, a cavity mode (c) appears which can be affected by the interactions with mode $s$. In the gap region, Fig. 3(a) shows the variation of the frequency of modes $s$ and $c$ with the thickness of a cavity located in the middle of a 8 BL-SL. While the frequency of mode $s$ remains almost independent of the cavity thickness $d_{c}$, the frequency of mode $c$ decreases monotonically with $d_{c}$. When the two frequencies of modes $c$ and $s$ approach each other, the interaction between their evanescent fields leads to an anticrossing of the dispersion curves [Fig. 3(a)]. For this observation a relatively small spatial separation between surface and cavity layers is required.

To address this predicted behavior experimentally, the thickness of the cavity layer located in the middle $\left(9^{P}\right)$ of the hybrid SLs (SL1, 3-5, Table I) was stepwise increased (from 42 to $140 \mathrm{~nm}$ ) while keeping the thickness of the surface layer roughly constant $\left(d_{s} \sim 50 \mathrm{~nm}\right)$. At large $d_{c}$, mode $c$ anticrosses mode $s$, but maintains the characteristic features of a cavity mode as proven by the displacement plot [Fig. 3(b)]. These findings conform well to theoretical work on the interaction of surface and cavity modes [7].

SLs terminated with the high elastic impedance layer exhibit no surface modes [27,37]. However, we show that if the top hard layer is significantly thicker than its precursor layer (inside the SL) the surface can again support a 


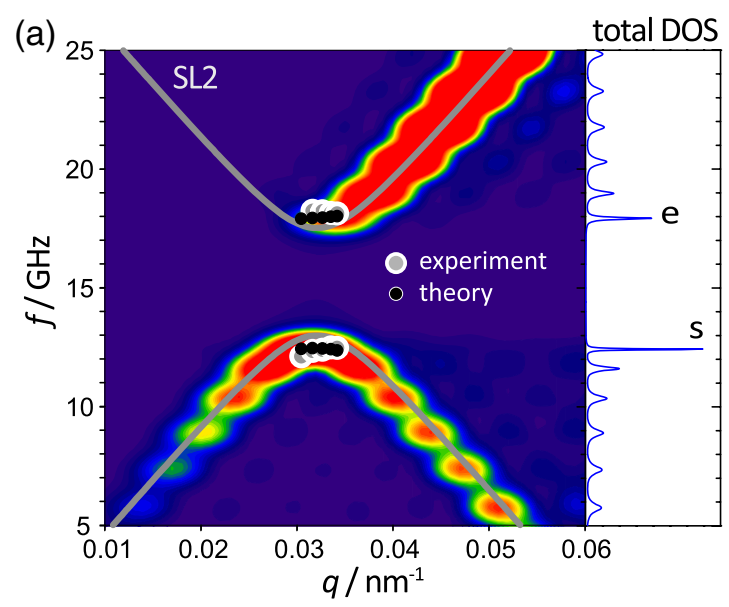

(b)

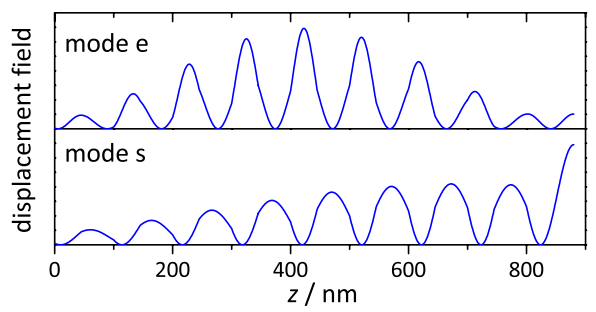

FIG. 2 (color online). (a) Dispersion relation and Brillouin intensity for SL2 with a thicker surface layer of PMMA compared to SL1. The solid lines indicate the band gap for the infinite SL. (b) The displacement field reveals the upper edge mode $(e)$ to be a surface avoiding mode while the lower band edge mode is merged with a surface mode $s$ with slow decay into the SL.

localized mode. Figure 4 displays the band diagram for SL6 bearing a $\mathrm{SiO}_{2}$ top layer with $d_{s} \sim 2 d_{\mathrm{SiO}_{2}}$. According to the associated DOS, two of the well-separated modes inside the gap are identified as the surface mode $(\sim 16.5 \mathrm{GHz})$ and the cavity mode $(\sim 15 \mathrm{GHz})$ based on the displacement field (not shown). This is clear evidence of the existence of the surface mode irrespectively of the rigidity of the top layer. In agreement with theoretical predictions [36] the existence of surface modes depends both upon the material and thickness of the top layer. Further, Fig. 4 shows the concurrent existence of mode $s$ induced by $\mathrm{SiO}_{2}$ at the surface and mode $c$ induced by the PMMA layer in the middle of SL6. Similar to Fig. 3, these modes may interact with each other inside the band gap when changing the thickness of PMMA and/or $\mathrm{SiO}_{2}$ layers.

Finally, in the semi-infinite SL7 (Fig. S4 in the Supplemental Material) the experimental peaks are in fact the sum over several DOS contributions [30]. Consequently, the mode dispersion lines up with the prediction (solid lines) for infinite SLs, while a flat mode due to the embedded cavities persists inside the band gap.

The phononic band structure of hybrid SLs is proven to be very sensitive to periodicity perturbations due to the (a)

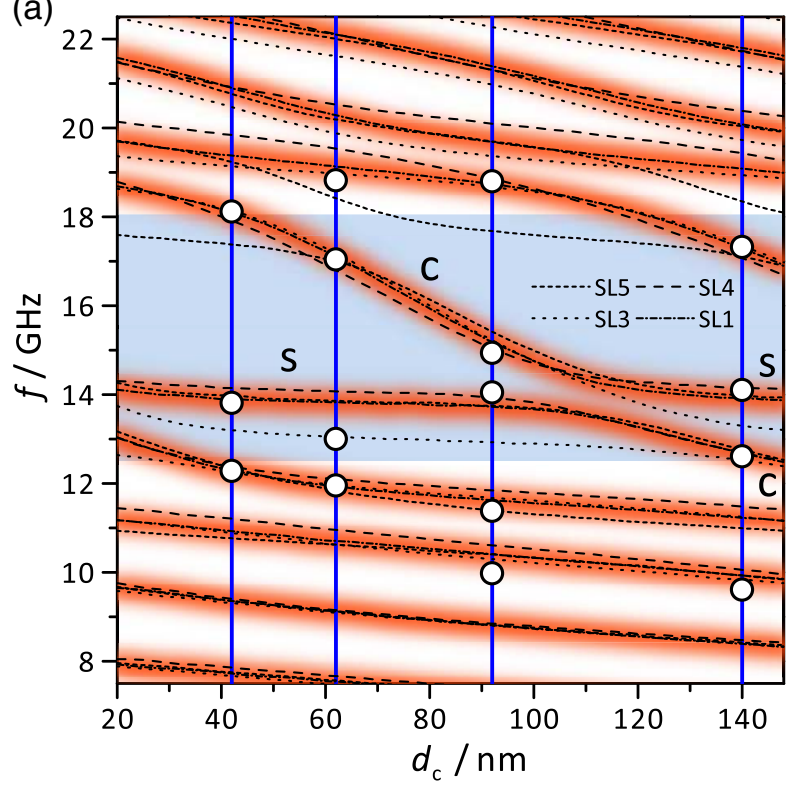

(b)

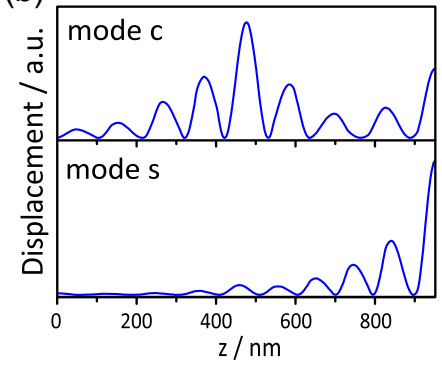

(c)

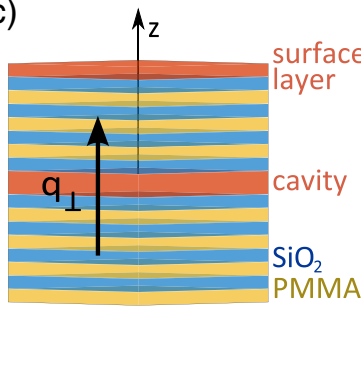

FIG. 3 (color online). (a) Mode dispersion around the Bragg gap as a function of cavity thickness. As the central PMMA layer is enlarged the cavity mode shifts inside the band gap and interacts with a surface mode for large cavity stacks. Experimental data are given by circles. The shaded region denotes the gap region of SL1. (b) Displacement field for modes $c$ and $s$. (c) Schematic indicating the position of the surface and cavity layers.

high symmetry breaking by introduction of defect layers. We presented the first unambiguous experimental evidence for the existence and significant interaction of surface and cavity modes in finite SLs supported by well-established theoretical predictions. The strong response to layer thickness and sequence opens pathways to phononic devices based on soft matter. Dynamic tuning of cavity modes and interaction with other defect modes under external stimuli (e.g., phase transformation using thermo- or chemiresponsive layers) could be used for detection applications. Finally, because of both acoustic and optical mismatch of the composing materials, hybrid SLs constitute a platform for new dual phononic-photonic (or phoxonic) structures for concurrent tuning of acoustic transmission in the $\mathrm{GHz}$ range and optical transmission in the visible by adjustment of layer thicknesses. For instance, sample SL1 displays an optical stop band around $290 \mathrm{~nm}$ (Fig. S5) [30]. 


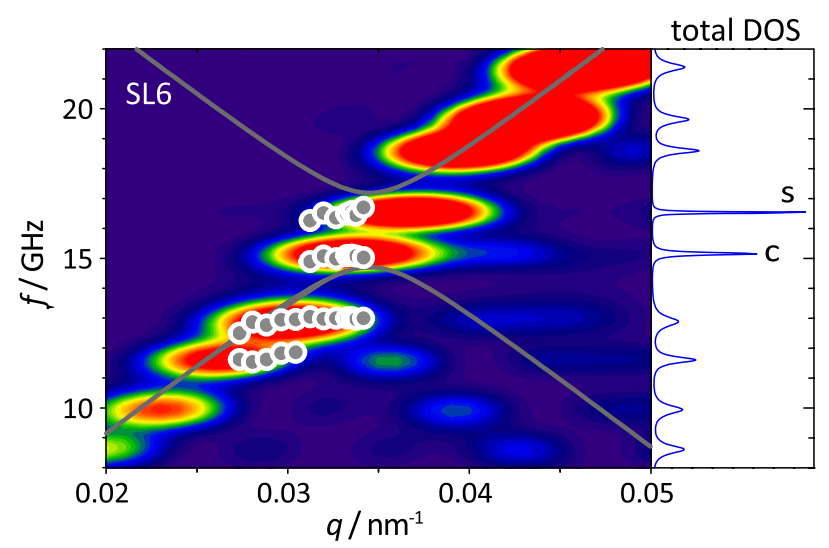

FIG. 4 (color online). Dispersion relation with Brillouin intensity and DOS for SL6 (with a thick $\mathrm{SiO}_{2}$ surface layer), which accommodates two well-separated modes in the band gap region, a surface $(s)$ and a cavity mode $(c)$. Experimental data are given by white circles.

DFG (Grants No. BU 1556/27 and No. IRTG 1404) and GSRT of Greece (ARISTEIA No. 285) are acknowledged for partial financial support. Three of the authors (EHEB, OEA and BDR) would like to thank the European Commission (EC) 7th Framework Programme (FP7), for support under the (IP) Project Reference No. 216176 (NANOPACK).

*Bahram.Djafari-Rouhani@univ-lille1.fr †fytas@mpip-mainz.mpg.de

[1] V. Narayanamurti, H. L. Störmer, M. A. Chin, A. C. Gossard, and W. Wiegmann, Phys. Rev. Lett. 43, 2012 (1979).

[2] M. S. Kushwaha, P. Halevi, L. Dobrzynski, and B. DjafariRouhani, Phys. Rev. Lett. 71, 2022 (1993).

[3] E. Yablonovitch, Phys. Rev. Lett. 58, 2059 (1987).

[4] R. Martinez-Salazar, J. Sancho, J. V. Sanchez, V. Gomez, J. Llinares, and F. Meseguer, Nature (London) 378, 241 (1995).

[5] Z. Liu, X. Zhang, Y. Mao, Y. Y. Zhu, Z. Yang, C. T. Chan, and P. Sheng, Science 289, 1734 (2000).

[6] T. Gorishnyy, C. K. Ullal, M. Maldovan, G. Fytas, and E. L. Thomas, Phys. Rev. Lett. 94, 115501 (2005).

[7] E. H. El Boudouti, B. Djafari-Rouhani, A. Akjouj, and L. Dobrzynski, Surf. Sci. Rep. 64, 471 (2009).

[8] Y. Pennec, J. O. Vasseur, B. Djafari-Rouhani, L. Dobrzyński, and P. A. Deymier, Surf. Sci. Rep. 65, 229 (2010).

[9] C. Colvard, R. Merlin, M. V. Klein, and A. C. Gossard, Phys. Rev. Lett. 45, 298 (1980).

[10] M. Trigo, A. Bruchhausen, A. Fainstein, B. Jusserand, and V. Thierry-Mieg, Phys. Rev. Lett. 89, 227402 (2002).

[11] H. T. Grahn, H. J. Maris, J. Tauc, and B. Abeles, Phys. Rev. B 38, 6066 (1988).
[12] H. J. Trodahl, P. V. Santos, G. V.M. Williams, and A. Bittar, Phys. Rev. B 40, 8577 (1989).

[13] V. Lemos, O. Pilla, M. Montagna, and C. de Souza, Superlattices Microstruct. 17, 51 (1995).

[14] N.-W. Pu and J. Bokor, Phys. Rev. Lett. 91, 076101 (2003).

[15] M. Trigo, T. A. Eckhause, M. Reason, R. S. Goldman, and R. Merlin, Phys. Rev. Lett. 97, 124301 (2006).

[16] Y. Ezzahri, S. Grauby, J. M. Rampnoux, H. Michel, G. Pernot, W. Claeys, S. Dilhaire, C. Rossignol, G. Zeng, and A. Shakouri, Phys. Rev. B 75, 195309 (2007).

[17] L. Belliard, A. Huynh, B. Perrin, A. Michel, G. Abadias, and C. Jaouen, Phys. Rev. B 80, 155424 (2009).

[18] N. D. Lanzillotti-Kimura, A. Fainstein, A. Huynh, B. Perrin, B. Jusserand, A. Miard, and A. Lemaître, Phys. Rev. Lett. 99, 217405 (2007).

[19] R. P. Beardsley, A. V. Akimov, M. Henini, and A. J. Kent, Phys. Rev. Lett. 104, 085501 (2010).

[20] P. Lacharmoise, A. Fainstein, B. Jusserand, and V. Thierry-Mieg, Appl. Phys. Lett. 84, 3274 (2004).

[21] M. Maldovan and E. L. Thomas, Appl. Phys. Lett. 88, 251907 (2006).

[22] S. R. Entezar and A. Namdar, Phys. Rev. A 80, 013814 (2009).

[23] B. Liang, X. S. Guo, J. Tu, D. Zhang, and J. C. Cheng, Nat. Mater. 9, 989 (2010).

[24] S.-M. Lee, D. G. Cahill, and R. Venkatasubramanian, Appl. Phys. Lett. 70, 2957 (1997).

[25] Y. K. Koh, Y. Cao, D. G. Cahill, and D. Jena, Adv. Funct. Mater. 19, 610 (2009).

[26] N. Gomopoulos, D. Maschke, C. Y. Koh, E. L. Thomas, W. Tremel, H. J. Butt, and G. Fytas, Nano Lett. 10, 980 (2010).

[27] D. Schneider, F. Liaqat, E. H. El Boudouti, Y. El Hassouani, B. Djafari-Rouhani, W. Tremel, H.-J. Butt, and G. Fytas, Nano Lett. 12, 3101 (2012).

[28] P. M. Walker, J.S. Sharp, A. V. Akimov, and A. J. Kent, Appl. Phys. Lett. 97, 073106 (2010).

[29] L. M. Goldenberg, V. Lisinetskii, and S. Schrader, Laser Phys. Lett. 10, 055808 (2013).

[30] See Supplemental Material at http://link.aps.org/ supplemental/10.1103/PhysRevLett.111.164301 for further information on fabrication, theoretical description of the BLS spectra and a complementary SL sample.

[31] J. He, B. Djafari-Rouhani, and J. Sapriel, Phys. Rev. B 37, 4086 (1988).

[32] P.X. Zhang, D. J. Lockwood, H. J. Labbé, and J.-M. Baribeau, Phys. Rev. B 46, 9881 (1992).

[33] M. Giehler, T. Ruf, M. Cardona, and K. Ploog, Phys. Rev. B 55, 7124 (1997).

[34] M. Trigo, A. Fainstein, B. Jusserand, and V. Thierry-Mieg, Phys. Rev. B 66, 125311 (2002).

[35] N. Combe, J. R. Huntzinger, and J. Morillo, Eur. Phys. J. B 68, 47 (2009).

[36] E. H. El Boudouti, B. Djafari-Rouhani, E. M. Khourdifi, and L. Dobrzynski, Phys. Rev. B 48, 10987 (1993).

[37] L. C. Parsons and G. T. Andrews, J. Appl. Phys. 111, 123521 (2012). 